\title{
Article \\ Cardiac Extracellular Matrix Hydrogel Enriched with Polyethylene Glycol Presents Improved Gelation Time and Increased On-Target Site Retention of Extracellular Vesicles
}

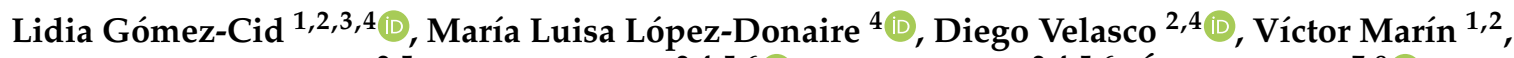

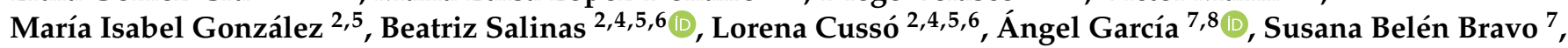 \\ María Eugenia Fernández-Santos 1,2,3,9 D, Carlos Elvira ${ }^{10}$, Johanna Sierra 2,11, Ester Arroba 2,11, \\ Rafael Bañares 2,9,11,12, Lilian Grigorian-Shamagian 1,2,3,9,* and Francisco Fernández-Avilés 1,2,3,9 (D)
}

check for updates

Citation: Gómez-Cid, L.; López-

Donaire, M.L.; Velasco, D.; Marín, V.; González, M.I.; Salinas, B.; Cussó, L.; García, Á.; Bravo, S.B.; Fernández-

Santos, M.E.; et al. Cardiac

Extracellular Matrix Hydrogel

Enriched with Polyethylene Glycol

Presents Improved Gelation Time and Increased On-Target Site Retention of Extracellular Vesicles. Int. J. Mol. Sci. 2021, 22, 9226. https://doi.org/ $10.3390 /$ ijms 22179226

Academic Editor: Frank Zaucke

Received: 30 July 2021

Accepted: 22 August 2021

Published: 26 August 2021

Publisher's Note: MDPI stays neutral with regard to jurisdictional claims in published maps and institutional affiliations.

Copyright: (c) 2021 by the authors. Licensee MDPI, Basel, Switzerland. This article is an open access article distributed under the terms and conditions of the Creative Commons Attribution (CC BY) license (https:// creativecommons.org/licenses/by/ $4.0 /)$.
1 Department of Cardiology, Hospital General Universitario Gregorio Marañón, 28009 Madrid, Spain; ligomezc@ing.uc3m.es (L.G.-C.); victor.magonzalez@alumnos.upm.es (V.M.); mariuge@fibhgm.org (M.E.F.-S.); francisco.fernandezaviles@salud.madrid.org (F.F.-A.)

2 Instituto de Investigación Sanitaria Gregorio Marañón, Hospital Gregorio Marañón, 28009 Madrid, Spain; divelasc@ing.uc3m.es (D.V.); migonzalez@hggm.es (M.I.G.); bsalinas@hggm.es (B.S.); lcusso@hggm.es (L.C.); johanna.sierra@iisgm.com (J.S.); ester.arroba@iisgm.com (E.A.); rbanares@ucm.es (R.B.)

3 CIBERCV, ISCIII, 28029 Madrid, Spain

4 Departamento de Bioingeniería e Ingeniería Aeroespacial, Universidad Carlos III de Madrid, 28911 Leganés, Spain; marisalop@ictp.csic.es

5 Unidad de Imagen Avanzada, Centro Nacional de Investigaciones Cardiovasculares (CNIC), 28029 Madrid, Spain

6 CIBERSAM, ISCIII, 28029 Madrid, Spain

7 Instituto de Investigación Sanitaria de Santiago de Compostela (IDIS), 15706 Santiago de Compostela, Spain; angel.garcia@usc.es (Á.G.); Susana.Belen.Bravo.Lopez@sergas.es (S.B.B.)

8 Center for Research in Molecular Medicine and Chronic Diseases (CIMUS), Universidad de Santiago de Compostela, 15706 Santiago de Compostela, Spain

9 Faculty of Medicine, Universidad Complutense de Madrid, 28040 Madrid, Spain

10 Institute of Polymer Science and Technology, CSIC, Juan de la Cierva 3, 28006 Madrid, Spain; celvira@ictp.csic.es

11 HepatoGastro Lab., Hospital General Universitario Gregorio Marañón, 28009 Madrid, Spain

12 CIBEREHD: ISCIII, 28029 Madrid, Spain

* Correspondence: lgrigorian@cibercv.es

\begin{abstract}
Stem-cell-derived extracellular vesicles (EVs) have demonstrated multiple beneficial effects in preclinical models of cardiac diseases. However, poor retention at the target site may limit their therapeutic efficacy. Cardiac extracellular matrix hydrogels (cECMH) seem promising as drugdelivery materials and could improve the retention of EVs, but may be limited by their long gelation time and soft mechanical properties. Our objective was to develop and characterize an optimized product combining cECMH, polyethylene glycol (PEG), and EVs (EVs-PEG-cECMH) in an attempt to overcome their individual limitations: long gelation time of the $\mathrm{CECMH}$ and poor retention of the EVs. The new combined product presented improved physicochemical properties $(60 \%$ reduction in half gelation time, $p<0.001$, and threefold increase in storage modulus, $p<0.01$, vs. cECMH alone), while preserving injectability and biodegradability. It also maintained in vitro bioactivity of its individual components (55\% reduction in cellular senescence vs. serum-free medium, $p<0.001$, similar to EVs and cECMH alone) and increased on-site retention in vivo (fourfold increase vs. EVs alone, $p<0.05$ ). In conclusion, the combination of EVs-PEG-CECMH is a potential multipronged product with improved gelation time and mechanical properties, increased on-site retention, and maintained bioactivity that, all together, may translate into boosted therapeutic efficacy.
\end{abstract}

Keywords: extracellular vesicles; hydrogel; extracellular matrix; drug delivery; polyethylene glycol; cardiac regenerative therapy 


\section{Introduction}

Regenerative and reparative therapies, although they seem promising in different cardiovascular diseases, present demanding requirements that are difficult to achieve and that limit their translation into the clinical scenario [1]. Regenerative products must be biocompatible and bioactive, preferably injectable in a unique dose, and adequately retained and degraded at the site of interest.

The main problems of cell products derive from their large variability and their lack of stability and standardization [2]. Some of the challenges seemed to be solved once it was clarified that most of their beneficial effects are caused by paracrine mediators, such as extracellular vesicles (EVs), which possess meaningful advantages as therapeutics vs. their parenteral cells [3,4]. In particular, EVs derived from cardiosphere-derived cells (CDCs) have shown potential to improve cardiac function in infarcted [5] and aged hearts [6], as well as antiarrhythmic effects [7]. However, EV retention at the target site remains as one of their main challenges [8].

The use of injectable biomaterials for cardiac regeneration, such as hydrogels, has also been explored. Those derived from cardiac extracellular matrix (cECMH) mimic the biophysical and topographical properties of the ECM and gel at physiological temperature. In addition, they improve cardiac function in preclinical models [9] and have shown safety in a phase I clinical trial [10]. However, slow gelation time, rapid degradation, and poor mechanical properties [11] are some of their physicochemical limitations. The combination of naturally derived hydrogels with synthetic materials, such as polyethylene glycol (PEG), seems promising [11] to make cECMH more suitable for therapeutic use.

Apart from providing structural support and favorable bioactivity on surrounding tissue, biomaterials can also be used for the delivery of small particles and/or cells to improve their retention at the injection site [12]. The combination of hydrogels with other bioactive products may solve some of the current limitations they possess individually, and enhance the therapeutic response with a synergistic effect [13]. However, specific designs for specific purposes will probably be needed.

The aim of this work was to develop, characterize, and evaluate in vitro and in vivo the suitability of a new product composed by EVs derived from CDCs embedded in a PEG-cECM hydrogel (EVs-PEG-cECMH) for its use as a regenerative product. Firstly, we explored the different physicochemical properties of the combined product (EVs-PEG-cECMH) vs. the cECMH alone, and how PEG, which can also be used as a purification method of EVs from conditioned medium, influenced the gelation time and the mechanical properties. Secondly, we confirmed that product combination into the EVs-PEG-cECMH did not negatively affect the bioactivity of the individual components and served to improve EV retention at the injection site compared to EV administration in the standard delivery vehicle.

\section{Results}

\subsection{Lyophylized cECM Retained Native ECM Proteins and SGAGs}

Decellularization of the myocardial matrix was confirmed by the absence of visible nuclei after DAPI staining. Blyscan assay on the lyophilized samples revealed a retained sulfated glycosaminoglycan (sGAG) content of $6.1 \pm 0.4 \mu \mathrm{g} / \mathrm{mg}$ of lyophilized cECM, of which $1.7 \pm 0.6 \mu \mathrm{g} / \mathrm{mg}$ was O-sulfated. Liquid chromatography-tandem mass spectrometry (LC-MS/MS) confirmed the presence of around 140 different proteins related to ECM and cardiac tissue. Figure 1 shows some of the most abundant proteins identified. Collagens of different types (I, VI, III, IV, and V) compose most of the cECM, but other relevant proteins involved in providing structure and promoting cell attachment and migration, such as fibrinogen, lumican, elastin, laminin, and fibronectin, are also present. 


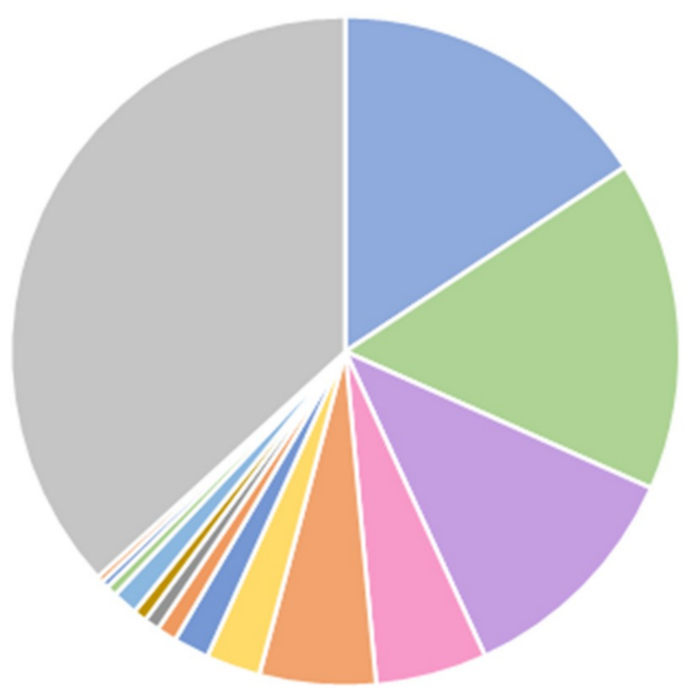

= Collagen I
= Collagen VI
= Fibrillar collagen NC1
= Collagen III
= Fibrinogen
= Collagen IV
= Collagen V
= Lumican
= Heparan sulfate proteoglycan
= Elastin
= Laminin
= Fibronectin
= Periostin
= Fibrillin-1
= Others

Figure 1. Proteins identified by liquid chromatography-tandem mass spectrometry (LC-MS/MS) in the lyophilized cardiac extracellular matrix (cECM) $(n=3)$.

2.2. cECM Hydrogels Incorporating EVs and PEG Have Shorter Gelation Times, Larger Fiber Diameter, and Improved Mechanical Properties While Remaining Injectable and Biodegradable

\subsubsection{Gelation Kinetics}

Turbidimetry measurements at $37^{\circ} \mathrm{C}$ showed an increase in optical density (OD) as the solutions gelled. After $90 \mathrm{~min}$, OD had reached a plateau in all the samples. The incorporation of PEG to the cECM solution significantly reduced the amount of time needed to reach the plateau, and, therefore, the gelation time (Figure $2 a$ ). While $t_{1 / 2}$ was $42 \pm 2 \mathrm{~min}$ for the control condition, when PEG was incorporated at concentrations of 3,6 , and $12 \mathrm{mg} / \mathrm{mL}$, the $t_{1 / 2}$ was reduced to $34 \pm 4,20 \pm 5$, and $9 \pm 2 \mathrm{~min}$, respectively $\left(\mathrm{R}^{2}=0.96, p=0.02\right)$. The reduction in $t_{1 / 2}$ with the incorporation of PEG was achieved due to a faster initiation of polymerization (shorter lag phase, $\mathrm{t}_{l a g}, \mathrm{R}^{2}=0.99, p<0.01$ ) and to an increased speed of gelation (slope of the OD change, $\mathrm{R}^{2}=0.83, p=0.08$ ). The relationship of $t_{1 / 2}, t_{l a g}$, and the slope of gelation with the PEG concentration is summarized in Figure $2 b$. As PEG concentration was increased, the turbidity of the gels (final OD value without normalization) also increased (data not shown). For PEG concentrations higher than shown $(16 \mathrm{mg} / \mathrm{mL})$, no significant change in OD was observed during the $90 \mathrm{~min}$, and the solution did not polymerize, even after $24 \mathrm{~h}$ of incubation at $37^{\circ} \mathrm{C}$. This suggested that higher PEG concentrations impede adequate gelation.

For the combined product, EVs isolated from conditioned medium [6,7] were incorporated into the hydrogels (EVs-PEG-cECMH), observing similar gelation kinetics to hydrogels with PEG (Figure 2a). They also presented reduced lag and gelation time $\left(t_{1 / 2}=13 \pm 3 \mathrm{~min}\right.$ ) and a higher speed of gelation (slope $\left.=0.022 \pm 0.009\right)$. The estimated PEG concentration in the combined EVs-PEG-cECMH product was $8 \pm 2 \mathrm{mg}$ of PEG per $1 \mathrm{~mL}$ of cECM solution.

\subsubsection{Fiber Diameter}

Hydrogel formulations incorporating PEG and EVs formed the characteristic nanofibrous structure with an increase in average fiber diameter, as shown and quantified in scanning electron microscopy (SEM) images (Figure 2c). Fiber density was not homogeneous across the gels, and the fibers tended to be randomly oriented in all the samples. However, fiber diameter was strongly correlated to the amount of PEG in the hydrogel $\left(\mathrm{R}^{2}=0.72, p<0.01\right)$. EVs-PEG-CECMH also presented an increased fiber diameter with respect to standard hydrogels $(100 \pm 20 \mathrm{~nm}$ vs. $60 \pm 20 \mathrm{~nm}, p<0.001)$. 


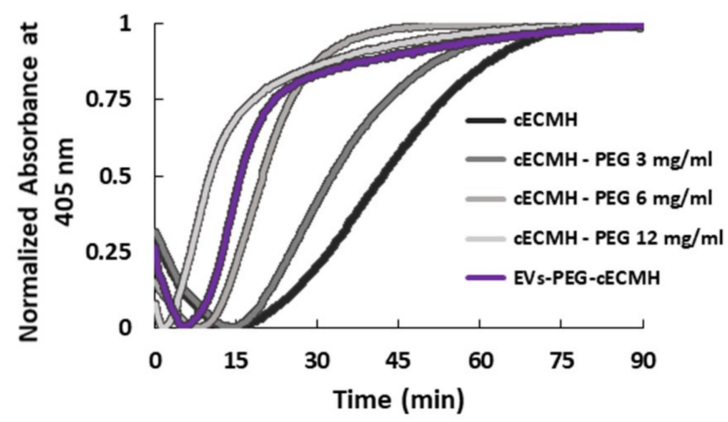

(a)
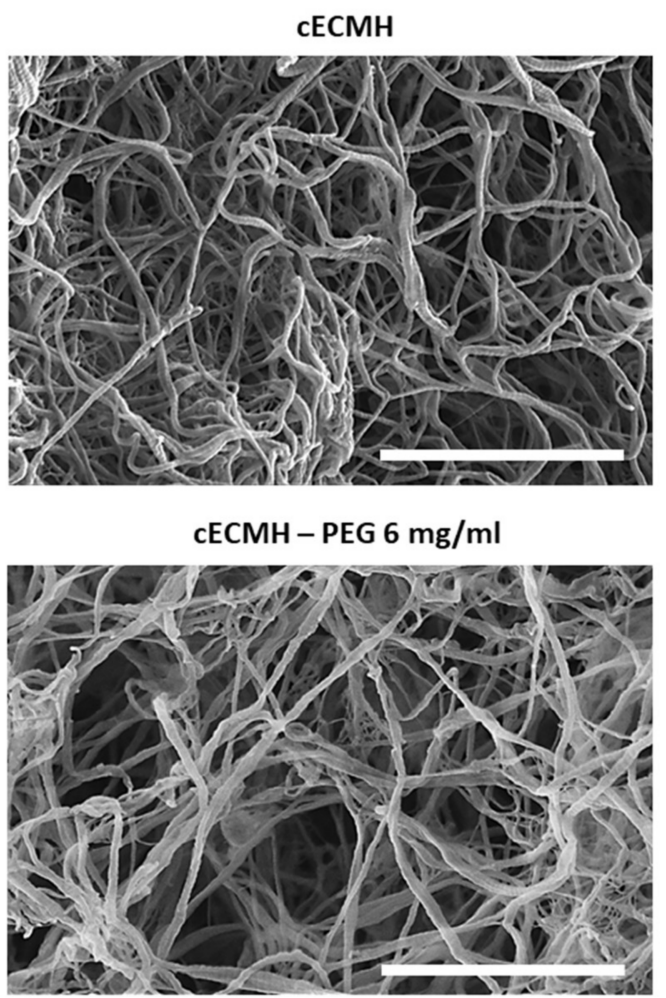

EVs-PEG-cECMH

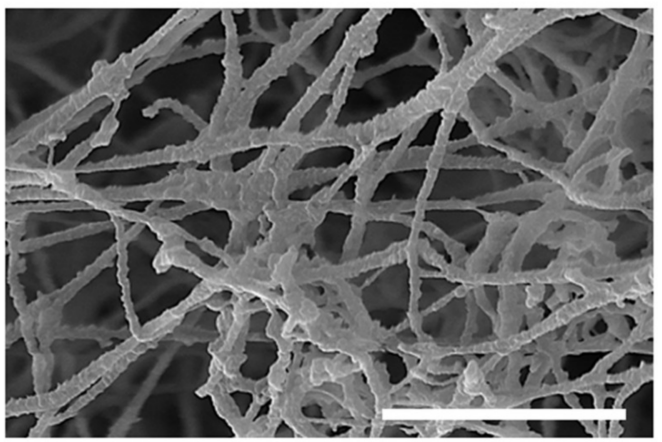

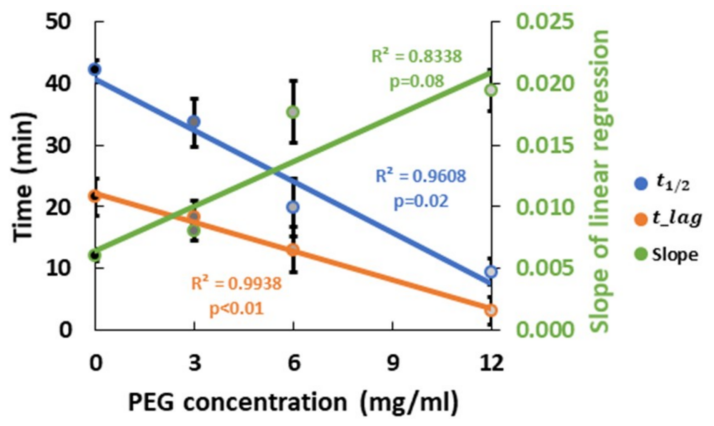

(b)
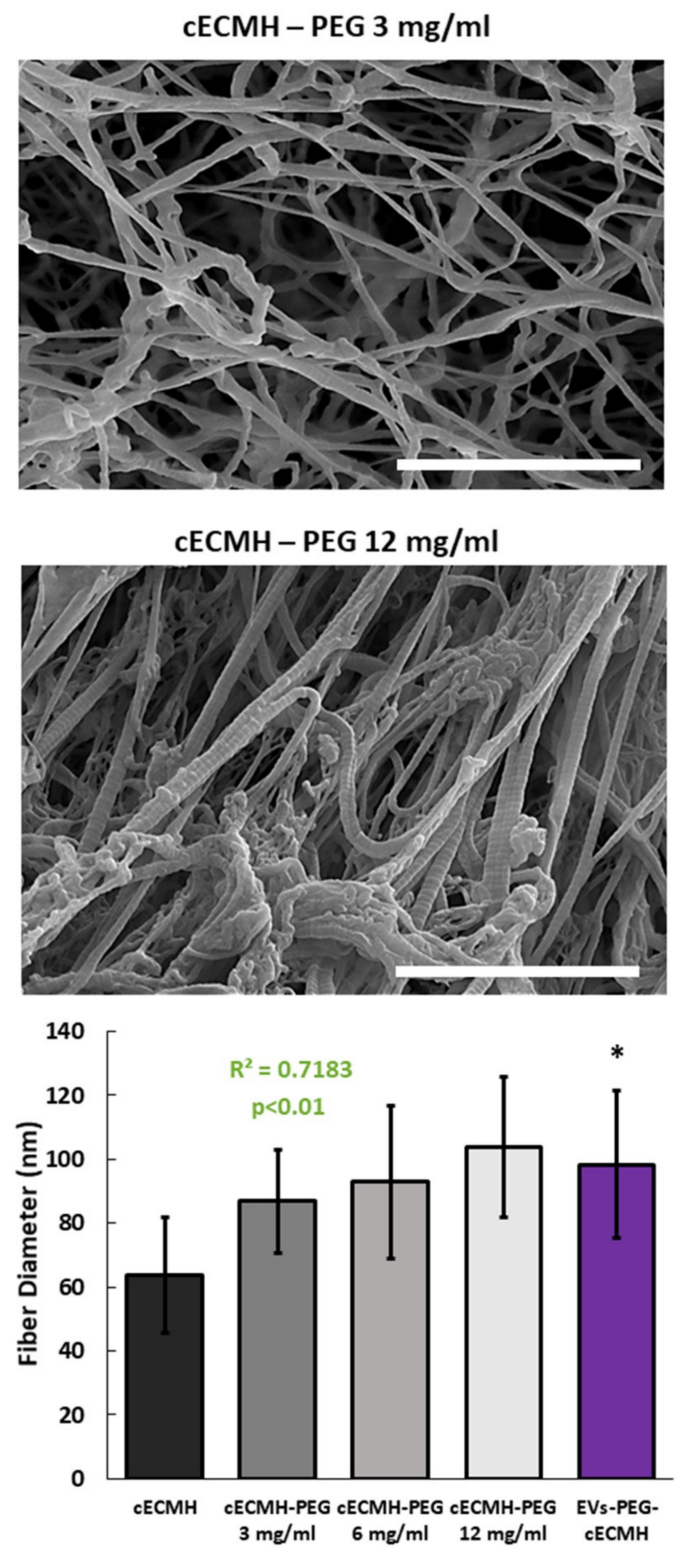

(c)

Figure 2. Gelation kinetics and fiber diameter. (a) Turbidimetric average results over time, with optical density normalized at $405 \mathrm{~nm}$ for the different formulations ( $n=3$, each in triplicate, for each condition). (b) Correlation between the PEG concentration and the half gelation time $\left(t_{1 / 2}\right)$, the lag time $\left(t_{l a g}\right)$, and the slope of the linear regression (speed of gelation) obtained from turbidimetry ( $n=3$, each in triplicate, for each condition). (c) Scanning electron microscopy (SEM) images of the different hydrogel formulations and their average fiber diameter ( $n=160$ fibers for each condition). Scale bars correspond to $2 \mu \mathrm{m}$. Squared Pearson's correlation coefficient for PEG concentration vs. fiber diameter and its significance 
$(p<0.01) .{ }^{*} p<0.001$ vs. cECMH. cECMH, cardiac extracellular matrix hydrogel; PEG, polyethylene glycol; EVs, extracellular vesicles.

\subsubsection{Injectability, Viscosity, Storage, and Loss Modulus}

All the formulations prepared remained injectable and with a viscosity suitable for their application through a Myostar injection catheter. In all the samples, the viscosity decreased as shear rate increased, as is characteristic of shear-thinning behavior (Figure 3a). This property is important for injectability, as well as for improved retention [14]. The formulation with the highest PEG concentration $(12 \mathrm{mg} / \mathrm{mL})$ presented inconsistent viscosity values (data not shown). These results seemed not to be reliable, since the very short gelation time of the samples with this concentration of PEG did not allow it to remain in soluble form during the viscosity measurement. For the remaining PEG concentrations ( 3 and $6 \mathrm{mg} / \mathrm{mL}$ ), the amount of PEG present did not relate with a change in the viscosity of the solutions at $0.16 \mathrm{~Hz}\left(\mathrm{R}^{2}=0.1, p>0.05\right)$, and the EVs-PEG-cECM did not present significant viscosity differences with respect to $c E C M$ alone (Figure $3 b$ ). Injection force measurements revealed that no additional force is required for the injection of $\mathrm{CECM}$ solutions incorporating PEG or the EVs-PEG-cECM compared to the injection of cECM solution alone (Figure 3c). However, the force required to inject any of the cECM solutions was significantly higher than the one required to inject the standard vehicle $(p<0.001$, phosphate-buffered saline, PBS).

The mechanical properties of the different hydrogel formulations are summarized in Figure 3d,e. The amount of incorporated PEG was directly related to an increase in the storage modulus of the derived hydrogels $\left(R^{2}=0.79, p<0.001\right)$. While higher PEG concentrations also lead to a significantly higher loss modulus, the correlation was weaker in comparison to the storage modulus $\left(\mathrm{R}^{2}=0.39, p<0.01\right)$. EVs-PEG-cECMH also presented a higher storage modulus than $c E C M H(15 \pm 5$ vs. $4.5 \pm 0.9 \mathrm{~Pa}, p<0.01)$, and a slightly higher loss modulus ( $1.5 \pm 0.5$ vs. $1.1 \pm 0.2, p>0.05)$.

\subsubsection{Degradation}

The ninhydrin assay (indicative of the amount of soluble amines) showed that PEG incorporation into the hydrogels did not affect their degradation rate, as there were no significant differences in OD at $570 \mathrm{~nm}$ between any of the formulations at the same time point ( $p>0.05$, Figure $3 f)$. All the samples presented significantly higher degradation after being incubated for $48 \mathrm{~h}$ with collagenase compared to $5 \mathrm{~h}$ of incubation $(p<0.05$, paired Student's $\mathrm{t}$-tests). The combined product (EVs-PEG-cECMH) presented the highest value of OD $(0.454 \pm 0.08$ after $5 \mathrm{~h}, 0.654 \pm 0.16$ after $24 \mathrm{~h}$, and $0.673 \pm 0.17$ after $48 \mathrm{~h})$ in the ninhydrin test. However, we did not consider this sample for comparison with the others because of the interference of the residual amines of the conditioned medium of the EVs in the ninhydrin test.

\subsection{The Combined Product of EVs-PEG-cECMH Maintains the Bioactivity of the Individual Components}

The bioactivity and potential beneficial effect of the PEG-cECMH with the embedded EVs was confirmed by evaluating the reduction in cardiac stromal cell (explant-derived cells, EDCs) senescence from two different donors in culture (this property of EVs derived from CDCs was proved in other studies [6]). As control groups for EVs-PEG-cECMH, we used EDCs incubated with serum-free media (SFM), EVs alone, or cECMH alone (Figure $4 a, b$ ). EVs and cECMH significantly reduced basal senescence (by around $40 \%$, $p<0.01)$ in the EDCs derived from both patients. No significant differences were detected between the EVs and the $\mathrm{CECMH}$ used alone. When using the combined product (EVsPEG-cECMH), basal senescence was further reduced (by $~ 55 \%, p<0.001$ ). This difference was also significant with respect to using EVs alone in one of the patients $(p<0.05)$. Images taken with optical microscopy in EDCs from patient 1 show a higher proportion of senescent EDCs (in blue) under basal conditions (SFM) than when exposed to EVs alone 
or cECMH alone, and a lower proportion of senescent cells when exposed to EVs-PEGcECMH (Figure 4c).

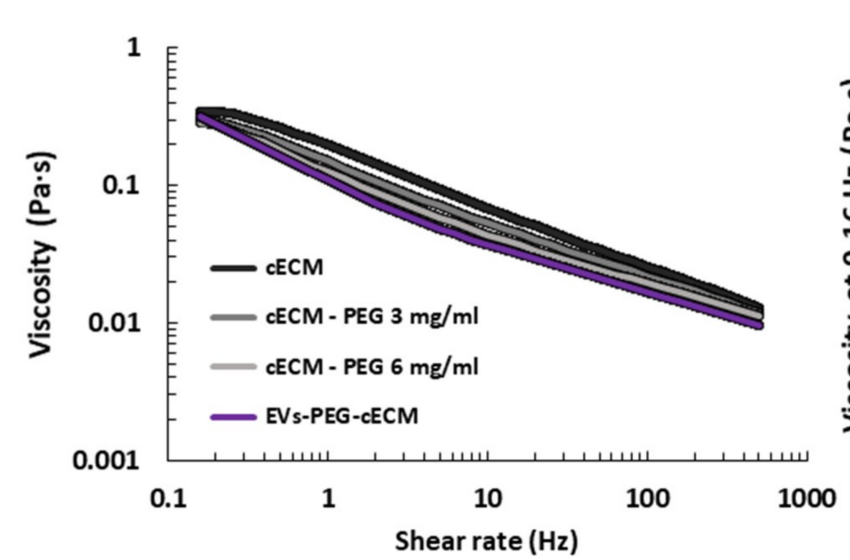

(a)

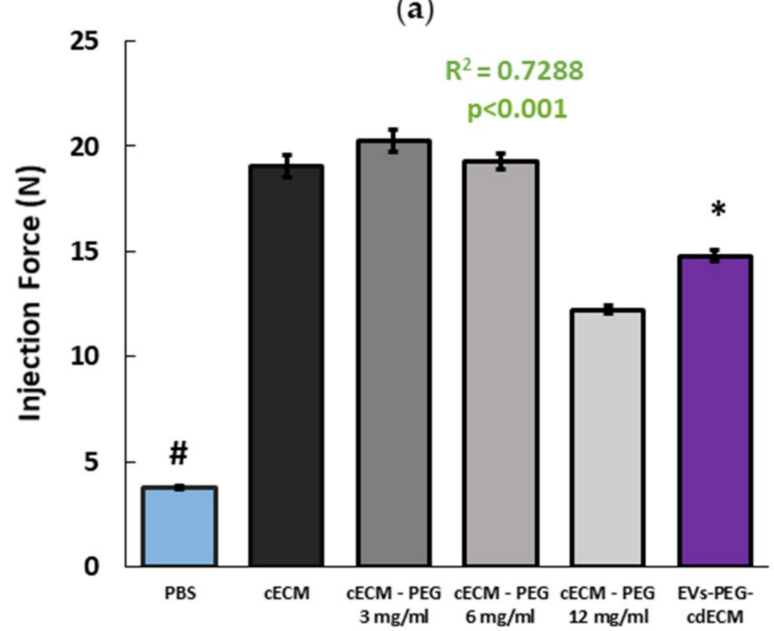

(c)

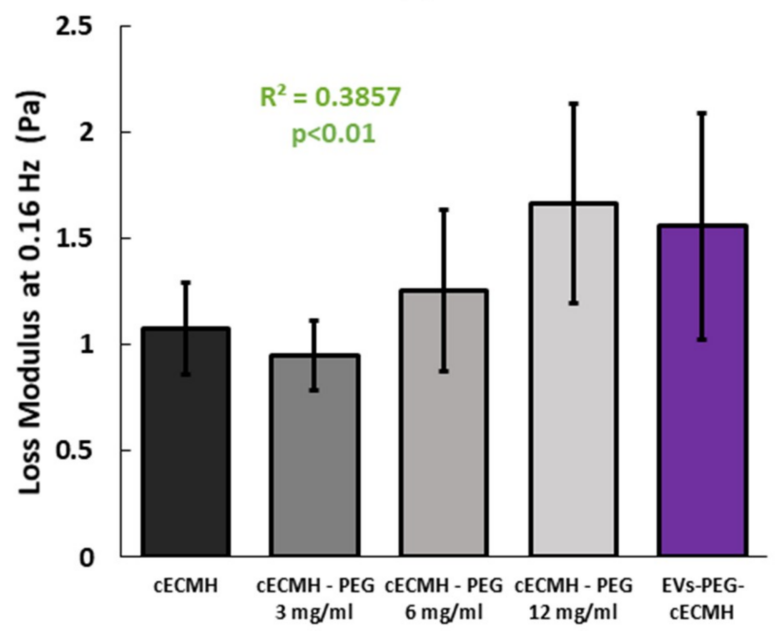

(e)

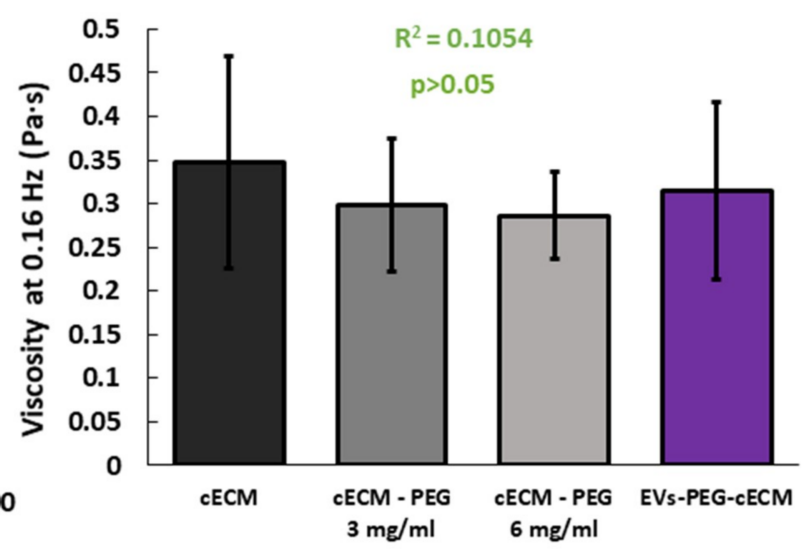

(b)

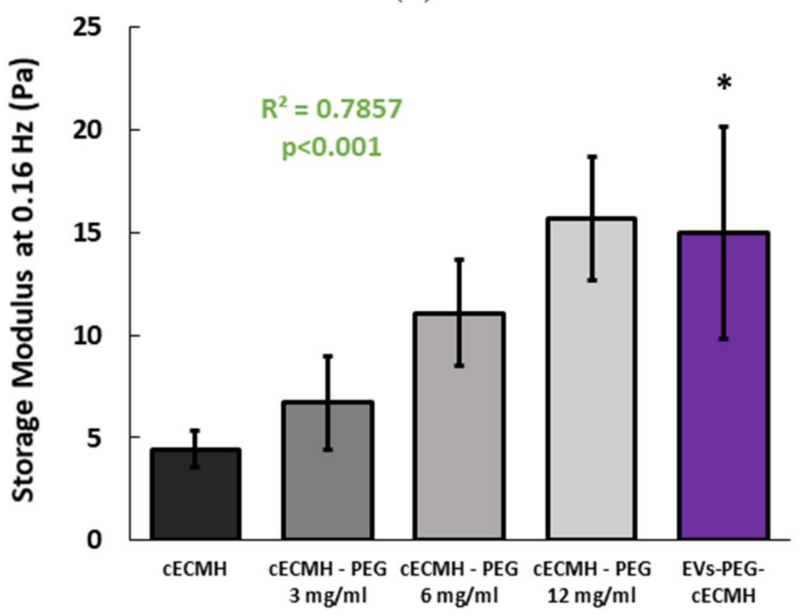

(d)

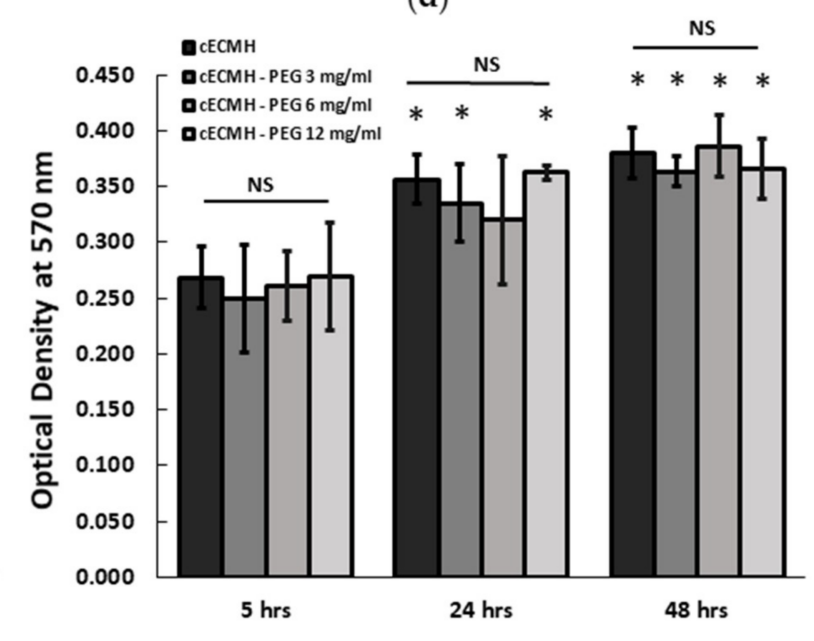

(f)

Figure 3. Mechanical properties and degradation of the cECMH alone, with PEG, and with PEG-isolated EVs. (a) Average viscosity of the different hydrogel solution formulations ( $n=3$ for each condition, each in triplicate). (b) Viscosity at $0.16 \mathrm{~Hz}(n=3)$. Squared Pearson's correlation coefficient for PEG concentration in the cECMH vs. viscosity at $0.16 \mathrm{~Hz}$ and its significance $(p>0.05)$. (c) Force required for injection $(n=3)$. Squared Pearson's correlation coefficient for PEG concentration in the cECMH vs. injection force and its significance $(p<0.001) .{ }^{*} p<0.001 \mathrm{vs}$. cECMH. $\# p<0.001$ vs. all other groups. (d) Storage modulus at $0.16 \mathrm{~Hz}(n=4)$. Squared Pearson's correlation coefficient for PEG concentration in 
the $\mathrm{cECMH}$ vs. storage modulus and its significance $(p<0.001) .{ }^{*} p<0.01 \mathrm{vs}$. cECMH. (e) Loss modulus at $0.16 \mathrm{~Hz}$ $(n=4)$. Squared Pearson's correlation coefficient for PEG concentration in the cECMH vs. loss modulus and its significance $(p<0.01)$ (f) Enzymatic degradation (soluble amines, $n=6$, each in triplicate). ${ }^{*} p<0.05$ with respect to their same group at 5 h. cECMH, cardiac extracellular matrix hydrogel; PEG, polyethylene glycol; EVs, extracellular vesicles.
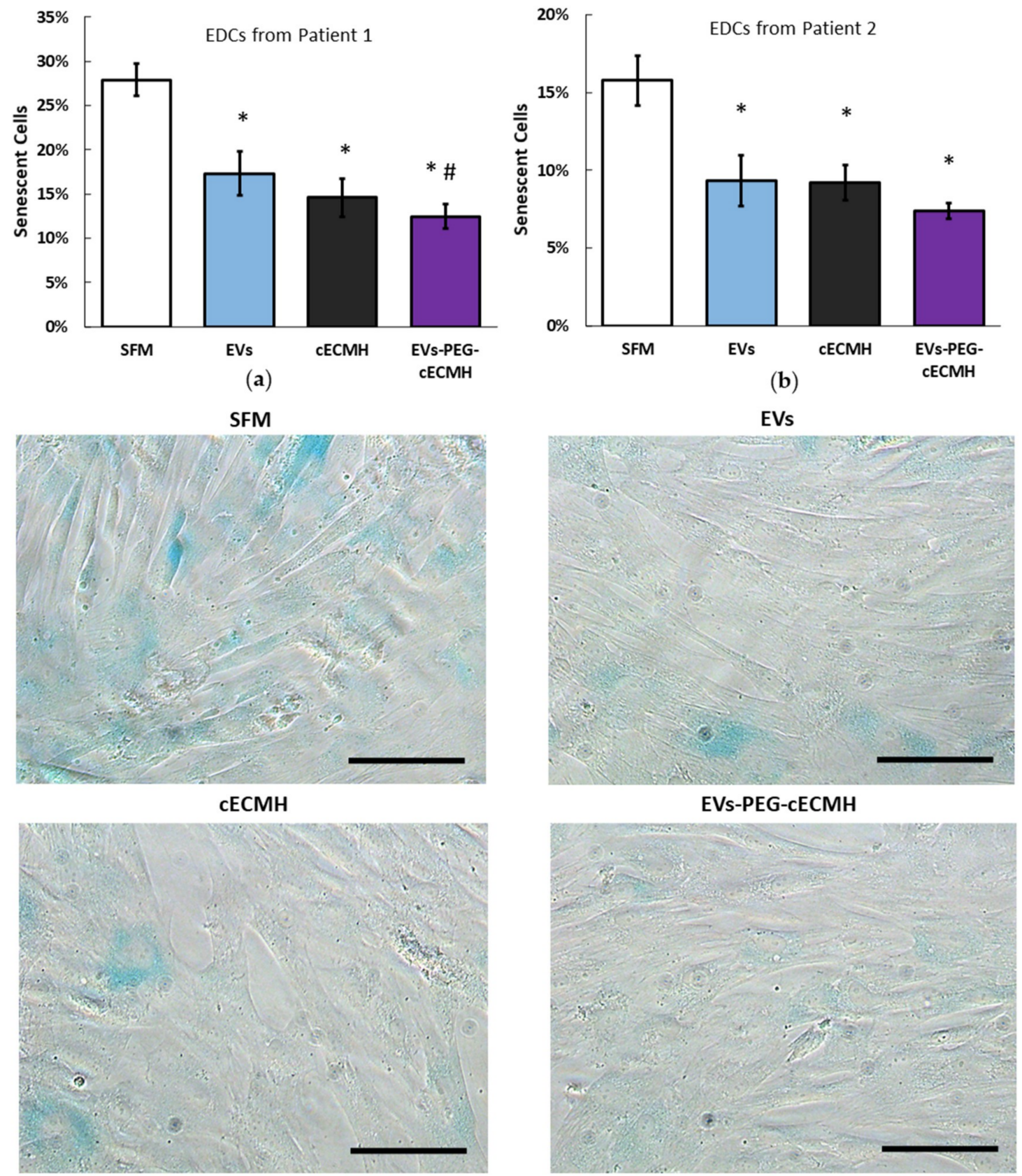

(c)

Figure 4. Bioactivity of EVs alone, $c E C M H$, and EVs-PEG-cECMH, measured as anti-senescent effect. (a) Percentage of senescent EDCs from patient 1 under basal conditions and under exposure to EVs alone, cECMH alone, or EVs-PEG-cECMH $(n=3)$. (b) Percentage of senescent explant-derived cells (EDCs) from patient 2 under the different conditions $(n=3)$. (c) Optical microscopy images from EDCs (senescent cells in blue) from patient 1 under the different conditions. ${ }^{*} p<0.01$ with respect to serum-free media (SFM). \# $p<0.05$ with respect to the EVs group. Scale bars correspond to $100 \mu \mathrm{m}$. cECMH, cardiac extracellular matrix hydrogel; PEG, polyethylene glycol; EVs, extracellular vesicles. 


\subsection{The Combined Product of EVs-PEG-cECMH Shows a Higher Local EV Retention In Vivo}

\subsubsection{EV Release}

Most EVs incorporated into the PEG-CECMH were cumulatively released during the first $48 \mathrm{~h}$ (Figure $5 \mathrm{a})$. After this time, there were no significant differences $(p>0.05)$ between the particles detected in control samples (EVs resuspended in SFM) and the cumulative number of particles released by the EVs-PEG-cECMH. During days 3 and 4, some particles continued to be released, but in considerably lower amounts. The number of particles detected in SFM alone (without EVs) or in medium exposed to cECMH or PEG-cECMH alone (without EVs embedded) was insignificant, confirming that particles detected in control samples and in samples from EVs-PEG-cECMH did correspond to the added EVs.

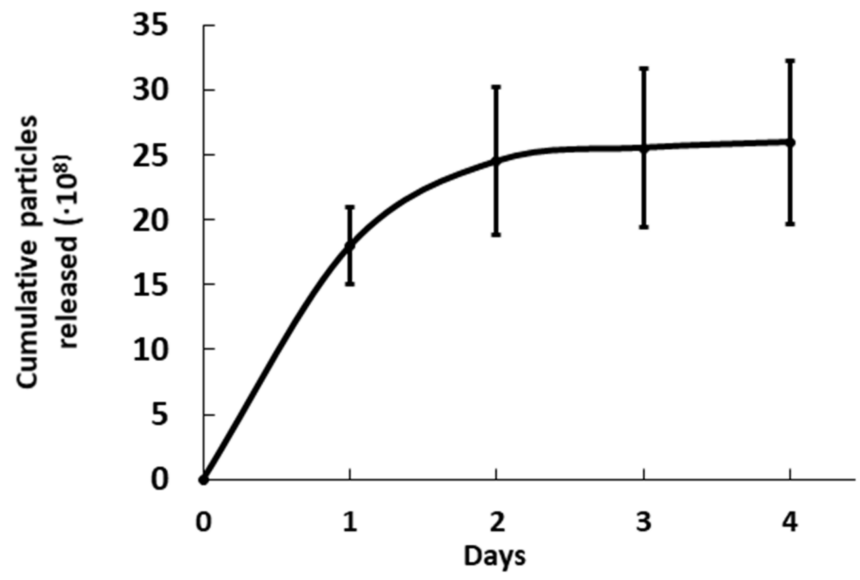

(a)
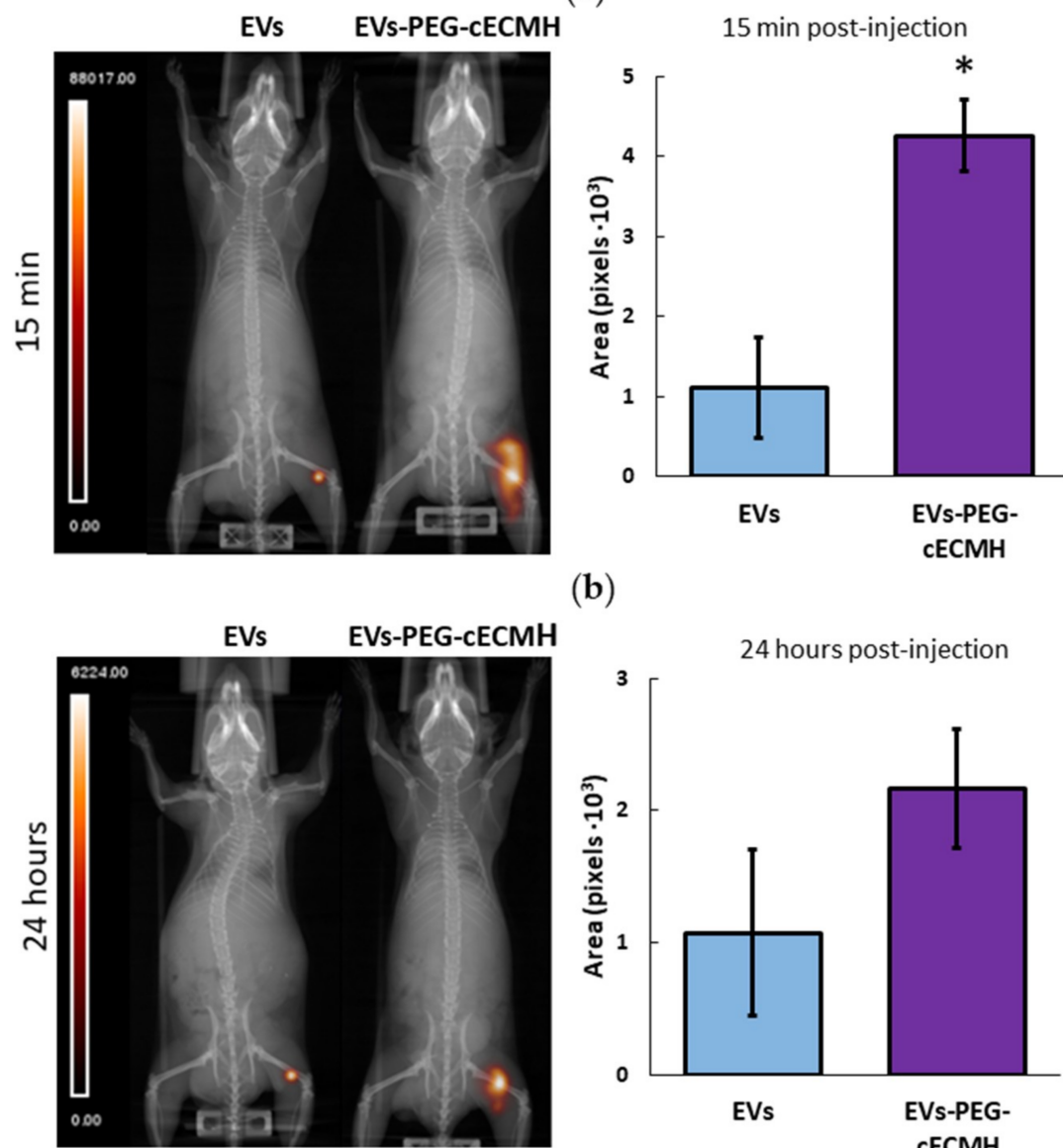

(b)

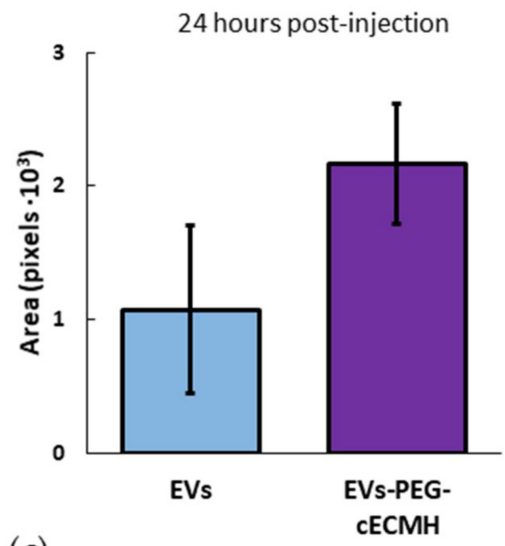

(c)

Figure 5. EV release from the EVs-PEG-cECMH and EV in vivo retention. (a) Number of cumulative 
EVs released into the medium from EVs-PEG-cECMH for 4 days ( $n=3$, each run in triplicate), evaluated using nanoparticle tracking analysis (NTA). The error bars were obtained by the standard deviation of the three different samples. After $48 \mathrm{~h}$ there were no significant differences $(p>0.05)$ with the number of particles detected in control samples. (b) Representative single-photon emission computed tomography-computed tomography (SPECT-CT) images and area of radioactively labeled EVs $(n=3)$ after in vivo injection of EVs resuspended in phosphate buffered saline (PBS) or in PEGcECM (EVs-PEG-cECM) $15 \mathrm{~min}$ and (c) $24 \mathrm{~h}$ post-injection. ${ }^{*} p<0.05$. cECMH, cardiac extracellular matrix hydrogel; PEG, polyethylene glycol; EVs, extracellular vesicles.

\subsubsection{In Vivo Retention}

Single-photon emission computed tomography-computed tomography (SPECT-CT) images of mice revealed a larger area of ${ }^{99 \mathrm{~m}}$ Tc radioactively labeled EVs at the subcutaneous injection site when administered embedded in the PEG-cECMH compared to their administration resuspended in PBS (Figure 5). The area of EVs was four times larger $(p<0.05)$ after 15 min post-injection when using the PEG-cECMH to deliver them. After $24 \mathrm{~h}$, the average area of concentrated EVs was twice as large when applied with the PEG-cECMH, but the difference was not significant $(p=0.1)$.

\section{Discussion}

According to our results, the combination of EVs-PEG-cECMH optimizes the features of its individual bioactive components and makes it more suitable in different therapeutical applications. EVs-PEG-cECMH maintained or significantly improved the physicochemical properties (particularly the gelation time), while not hindering injectability and degradation vs. cECMH alone. PEG at low concentrations, which, in fact, can be used to isolate EVs from conditioned medium, was responsible for these differences. In addition, the EVs are progressively released from the EVs-PEG-cECMH and are better retained at the injection site in vivo when administered in the EVs-PEG-cECMH compared to EVs administered in the standard vehicle (PBS). The combination of the products reduces cellular senescence, maintaining the bioactive properties of cECMH and EVs alone.

Although the incorporation of low-molecular-weight PEG up to a certain concentration $(12 \mathrm{mg} / \mathrm{mL})$ proportionally improved the gelation properties of the $\mathrm{CECMH}$, higher PEG concentrations impeded gelation. cECMH have collagen as their main component, so they share some similarities in the gelation kinetics with collagen hydrogels [18]. During the lag phase, collagen nucleation occurs by forming triple helices. Later, during the gelation phase, these assemble into ordered structures to form fibrils [19]. The addition of PEG seems to speed up the nucleation phase and favor collagen fibril formation during gelation. This more rapid formation could be responsible for the larger fiber diameter [20] and the higher turbidity of the gels [19]. Larger fiber diameter could also result from PEG entangled in collagen fibers, similar to hyaluronic acid chains incorporated into a collagen network without crosslinking [21]. Other studies have incorporated PEG into cECM or collagen hydrogels to tailor their material properties $[20,22]$. However, these studies incorporate multi-armed PEG with functionalized groups that crosslink with the collagen network or modified PEGs that require external triggers for polymerization, such as UV light. The addition of functionalized multi-armed PEGs at high concentrations $(12-24 \mathrm{mg} / \mathrm{mL})$ still allows the formation of gels at $37^{\circ} \mathrm{C}$ without external triggers and the tuning of mechanical properties and degradation, but they do not improve the gelation time [20]. Slow gelation times, which can increase tissue necrosis, have been highlighted as a main drawback of ECM hydrogels [11].

Mechanical properties of the ECM hydrogels are also improved with the incorporation of PEG, while injectability and biodegradation are maintained. The mechanical properties of cECMH alone are considered insufficiently robust for providing prolonged mechanical support in the injured heart, where they are subjected to significant strain and contraction [11]. In addition, these properties influence cell fate [23] and migration [24]. 
PEG-CECMH or EVs-PEG-cECMH present a significantly higher storage modulus, making them more suitable for cardiac applications than $\mathrm{CECMH}$ alone. The reduction in the gelation time and the increase in the elasticity of the cECMH after gelation did not negatively affect the viscosity of the liquid form and their injectability through the Myostar catheter. Even the solutions with reduced gelation time could be uniformly injected and did not clog the catheter, an essential property for cardiac applications [25]. In fact, when adding the $\mathrm{EVs}$, the force required for injection was considerably lower. Moreover, the incorporation of PEG with the method presented here (linear PEG without functionalized groups that react with amines in collagen) does not influence the cECMH biodegradation rate, which in vivo studies have shown to completely degrade within 14-28 days post-injection [9].

Hydrogels as vehicles to deliver EVs have already shown potential for improved in vivo retention and boosted cardiac function [15-17]. Applications where fast gelation time becomes especially important (e.g., to retain bioactive molecules at the site of injection [11], embed cells, or create 3D tissue-like structures [26]) while maintaining injectability and natural tissue environment may potentially benefit from the improved physicochemical properties of PEG incorporation into ECM hydrogels. The combined product presented here (EVs-PEG-CECMH), with an estimated PEG concentration of $8 \pm 2 \mathrm{mg}$, (matching the results obtained in the physicochemical analysis, in which the combined product presented average values between the cECMH with PEG at 6 and $12 \mathrm{mg} / \mathrm{mL}$ ), benefits from these improved physicochemical properties.

EVs administered in vivo in the PEG-cECM solution also achieved the goal of improved retention at the target site when compared to EVs administered with the standard vehicle (PBS). This is probably because the fast gelation time of the combined product prevents the rapid absorption of the subcutaneously injected EVs into the bloodstream right after the injection. According to nanoparticle tracking analysis (NTA) measurements, EVs encapsulated in the PEG-CECMH are mostly released during the first $48 \mathrm{~h}$, which is in line with other studies [27]. In addition, SEM images of the EVs-PEG-cECMH show rougher collagen fibers with respect to $\mathrm{CECMH}$ or PEG-CECMH alone. This phenomenon has also been observed in other studies, where this roughness has been related to embedded proteins and vesicles [28]. After several days of soaking the hydrogels, as the particles were released to the medium, the fibers started to look smoother. Accordingly, our in vivo experiment showed that, although a high proportion of the EVs are probably released from the PEG-cECMH during the first day, a large amount of EVs still remain encapsulated at the injection site after 24-h.

Both the cECMH [9] and the EVs [5] individually have shown favorable effects for the treatment of cardiac pathologies in preclinical models. While EVs have been shown to halt proinflammatory and profibrotic pathways and induce angiogenesis [5], the ECM hydrogels provide mechanical support and an environment with a structure and protein composition close to the native cardiac tissue [9]. Moreover, ECM hydrogels promote cellular influx and their degradation products favor cellular migration, proliferation [29], and angiogenesis [30]. In this study, despite EVs and the cECMH individually showing significant (and similar) anti-senescent properties in cardiac stromal cells from different human donors, this effect tended to be enhanced when both products were used together, indicating a possible synergistic effect of both products.

Despite the EVs embedded in PEG-cECMH showing promising characteristics for its use in cardiac regenerative applications, future studies should confirm if the improved physicochemical properties of the combined product (provided by the PEG), the bioactivity, and the enhanced in vivo EV retention vs. the use of the individual components alone translate into improved functionality in an animal model. Furthermore, mechanical properties of the PEG-cECMH, although improved with the addition of PEG, are still far from mimicking that of the native ECM. In this study, EVs were tracked in vivo only for $24 \mathrm{~h}$, and our in vitro tests demonstrated that most particles are released during the first $48 \mathrm{~h}$. Presumably, a longer liberation time of the EVs from the PEG-cECMH could increase the efficacy of the product, mimicking repeated dosing. Labeling the EVs with other molecular 
methods (such as with optical probes), would allow in vivo tracking of the EVs for longer. This could help determine for how long the EVs can remain at the target site after being injected in the PEG-cECMH. To further improve and optimize these properties, it becomes necessary to explore modifications in the hydrogel composition. For example, modifying the ECM concentration and incorporating additional synthetic materials could contribute to the tailoring of mechanical and degradation properties [18,20], as well as pore size [31] and EV release rate.

Regardless of the limitations cited above, here we show that the delivery of EVs isolated with PEG and embedded in cECMH offer a minimally invasive, injectable therapeutical product with a fast thermosensitive response at physiological temperature. The product can provide an environment with proteins naturally present in the myocardium and with mechanical and structural support, while it enables the retention and release of the EVs at the target site. The combined product (EVs-PEG-cECMH) presents bioactivity and is biodegradable. As EVs-PEG-CECMH solves some of the current limitations of this type of biological therapy, it can potentially be used in different regenerative medicine applications.

\section{Materials and Methods}

\subsection{EDCs, CDCs, and Derived Extracellular Vesicle Isolation}

EVs used as therapeutic product were isolated from human CDCs, as previously described [6,7]. Briefly, cardiac biopsies from patients undergoing cardiac surgery were processed to obtain 1-2 mm explants. EDCs colonized the plate after approximately 16 days. EDCs could be further cultured on ultra-low-attachment plates for $72 \mathrm{~h}$ to form cardiospheres. After three further passages in standard plates, CDCs were left in FBS-free IMDM (SFM) for 15 days to secrete EVs into the medium. The conditioned medium was filtrated using a $0.45 \mu \mathrm{m}$ filter and ultraconcentrated with Centricon-Plus 70 Centrifugal Filter with 3-kDa cut-off frequency. EVs were quantified by NTA ( Nanosight NS300, Malvern Paranalytical, Malvern, UK) and with Bradford assay after lysis and protease inactivation. The mean EV size obtained by the NTA software was $170 \mathrm{~nm} \pm 20 \mathrm{~nm}$, with a maximum particle size of $450 \mathrm{~nm}$. For experiments, EVs were incubated overnight at $4{ }^{\circ} \mathrm{C}$ in $4 \% w / v$ polyethylene glycol (PEG), precipitated by centrifugation at $1500 \times g$ for $30 \mathrm{~min}$, and resuspended in the corresponding medium (SFM or CECMH) at a concentration of $5 \mathrm{mg} / \mathrm{mL}$, unless otherwise stated. Considering the $4 \%$ w/v PEG concentration in the conditioned medium, the size of the pellet containing the EVs $(150-250 \mu \mathrm{L})$ and the volume of cECM in which the pellet was resuspended $(1 \mathrm{~mL})$, the estimated PEG concentration in the combined product of EVs-PEG-cECM solution was $8 \pm 2 \mathrm{mg} / \mathrm{mL}$.

\subsection{Porcine Myocardial Matrix Decellularization, Lyophilization, and Characterization}

Porcine myocardial matrix was decellularized with slight modifications from a previous protocol from our group [32]. The heart from six euthanized minipigs (6.5 months old on average, 3 males) were immediately extracted, cleaned from fat, valves, and fibrotic regions, and the myocardium was cut into thin slices ( $1 \mathrm{~mm}$ thick). The slices were individually decellularized in a distilled water solution at $1 \% \mathrm{w} / \mathrm{v}$ of sodium dodecyl sulfate (SDS) under constant shaking for 48-72 h, and then washed in distilled water for another $72 \mathrm{~h}$. All the solutions were changed daily. The slices were then disinfected for $30 \mathrm{~min}$ in peracetic acid at $1 \%$ and kept in phosphate-buffered saline (PBS) with 1\% penicillin/streptomycin (Gibco) at $4{ }^{\circ} \mathrm{C}$ until lyophilization. Three slices from different animals (decellularized and non-decellularized) were frozen in Tissue O.C.T., cut into $4 \mu \mathrm{m}$ slices, and stained with DAPI to confirm decellularization. This decellularization protocol has been previously shown in our group to yield less than $50 \mathrm{ng}$ of DNA content per mg of tissue [32]. The lyophilized samples (in a Telstar, Lioalfa-6 lyophilizer, Munich, Germany) were later cut into small pieces (around $1 \mathrm{~mm}$ size), mixed, and stored at $-20^{\circ} \mathrm{C}$ until used for hydrogel preparation. sGAG content (total and O-sulfated) and proteins present in the lyophilized matrix were confirmed in 3 samples by using the Blyscan assay (Biocolor Ltd., 
Carrickfergus, UK) and by LC-MS/MS (in a Triple TOF 6600, Sciex, Old Connecticut Path Framingham, MA, USA), respectively.

The LC-MS/MS analysis was made using a previously standardized method by our group [33,34]. An equal amount of protein from the samples was loaded on a $10 \%$ SDSPAGE gel. The run was stopped as soon as the front had penetrated $3 \mathrm{~mm}$ into the resolving gel $[35,36]$. The protein band was visualized by Sypro Ruby fluorescent staining (Lonza, Basel, Switzerland), excised, and subjected to in-gel, manual tryptic digestion following the protocol described previously by our group $[33,34]$. Peptides were extracted by performing three 20 -min incubations in $40 \mu \mathrm{L}$ of $60 \%$ acetonitrile dissolved in $0.5 \% \mathrm{HCOOH}$. The resulting peptide extracts were pooled, concentrated in a SpeedVac, and stored at $-20^{\circ} \mathrm{C}$.

Digested peptides were separated using reverse phase chromatography. Gradient was developed using a micro liquid chromatography system (Eksigent Technologies nanoLC 400, Sciex, Framingham, MA, USA) coupled to a high-speed Triple TOF 6600 mass spectrometer (Sciex, Framingham, MA, USA) with a micro flow source. The analytical column used was a silica-based reversed phase column YMC-TRIART C18 $150 \times 0.30 \mathrm{~mm}$, with a $3 \mathrm{~mm}$ particle size, and $120 \AA$ A pore size (YMC Technologies, Teknokroma, Sciex, Framingham, MA, USA). The trap column was a YMC-TRIART C18 (YMC Technologies, Teknokroma, Sciex, Framingham, MA, USA) with a $3 \mathrm{~mm}$ particle size and $120 \AA$ A pore size, switched on-line with the analytical column. The loading pump delivered a solution of $0.1 \%$ formic acid in water at $10 \mu \mathrm{L} / \mathrm{min}$. The micro-pump provided a flow rate of $5 \mu \mathrm{L} / \mathrm{min}$ and was operated under gradient elution conditions, using $0.1 \%$ formic acid in water as mobile phase A, and $0.1 \%$ formic acid in acetonitrile as mobile phase B. Peptides were separated using a 90 -minute gradient ranging from $2 \%$ to $90 \%$ mobile phase B (mobile phase A: $2 \%$ acetonitrile, $0.1 \%$ formic acid; mobile phase B: $100 \%$ acetonitrile, $0.1 \%$ formic acid). Injection volume was $4 \mu \mathrm{L}$.

Data acquisition was carried out in a TripleTOF 6600 System (Sciex, Framingham, MA, USA) using a data-dependent workflow. Source and interface conditions were as follows: ion spray voltage floating (ISVF) $5500 \mathrm{~V}$, curtain gas (CUR) 25, collision energy (CE) 10, and ion source gas 1 (GS1) 25. The instrument was operated with Analyst TF 1.7.1 software (Sciex, Framingham, MA, USA). Switching criteria were set to ions greater than the mass to charge ratio $(m / z) 350$ and smaller than $m / z 1400$, with charge state of 2-5, mass tolerance $250 \mathrm{ppm}$, and an abundance threshold of more than 200 counts (cps). Former target ions were excluded for $15 \mathrm{~s}$. The instrument was automatically calibrated every $4 \mathrm{~h}$ using as external calibrant tryptic peptides from pep Cal Mix (Sciex, Framingham, MA, USA).

After MS/MS analysis, data files were processed using ProteinPilotTM 5.0.1 software from Sciex, which uses the algorithm ParagonTM for database search and ProgroupTM for data grouping. Data were searched using a Sus Scrofa specific Uniprot database. False discovery rate was performed using a non-lineal fitting method, displaying only those results that reported a $1 \%$ global false discovery rate or better $[37,38]$. For the plot in Figure 1, relative abundance of each protein was determined from the number of peptides identified, corrected by the size of the protein (in $\mathrm{KDa}$, from Uniprot).

\subsection{Cardiac Extracellular Matrix Hydrogel (cECMH) Synthesis}

Hydrogels from the lyophilized decellularized $\mathrm{CECM}$ were prepared fresh for each experiment, with slight modifications from previous protocols [39]. The lyophilized myocardial matrix was solubilized with pepsin (P6887, Sigma-Aldrich, at $1 \mathrm{mg} / 10 \mathrm{mg}$ of lyophilized matrix) in $0.01 \mathrm{M} \mathrm{HCl}(1 \mathrm{~mL} / 10 \mathrm{mg}$ of matrix) under constant stirring for $72 \mathrm{~h}$, at which point no solid particles were visible. The solubilized cardiac matrix solution was placed on ice and adjusted to $\mathrm{pH} 7.4$ by progressive addition of $0.1 \mathrm{M} \mathrm{NaOH}$, and to physiological salt concentration (PBS 1x) by addition of $1 / 9$ of the volume of PBS 10x. For hydrogel formulations incorporating PEG, (molecular weight $=8000$, P5414, Sigma-Aldrich, at $3,6,12$, or $16 \mathrm{mg} / \mathrm{mL}$ in the final solution), the corresponding amount of polymer was dissolved in the PBS 10x, sterile filtered, and added to the solution. All formulations were brought to a final concentration of $8 \mathrm{mg}$ of solubilized cardiac matrix per $\mathrm{mL}$ of solution 
with the addition of PBS 1x. The solution was centrifuged at $4000 \mathrm{rpm}$ and $4{ }^{\circ} \mathrm{C}$ for $5 \mathrm{~min}$ to remove any remaining insoluble particles (if any) and kept at $4{ }^{\circ} \mathrm{C}$ until used (maximum $24 \mathrm{~h})$.

\subsection{Gelation Kinetics}

Gelation kinetics from three different batches of the control hydrogels, with PEG at 3, 6, 12, and $16 \mathrm{mg} / \mathrm{mL}$, and with EVs prepared as described above, were studied by turbidimetry as previously published $[18,28]$. A total of $100 \mu \mathrm{L}$ of each solution in triplicate were placed in a 96-well plate, and the OD at $405 \mathrm{~nm}$ and $37{ }^{\circ} \mathrm{C}$ was measured every $15 \mathrm{~s}$ with a SynergyTM HTX Multi-Mode Microplate Reader (BioteK, Winooski, VT, USA) during $90 \mathrm{~min}$ (when a plateau in all samples had been reached). OD values were averaged and normalized for each group. From the normalized plot, the half gelation time $\left(t_{1 / 2}\right.$, at which normalized OD was 0.5$)$, the lag phase $\left(t_{\text {lag }}\right.$, at which the linear fit in the linear region of the plot was zero), and the speed of gelation (S, slope of the linear fit) were compared among the different groups.

\subsection{Rheometry and Injectability}

Viscosity and rheology measurements were performed in a TA Instruments AR-G2 rheometer (New Castle, DE, USA) on samples with the different conditions (cECMH, and cECMH with PEG at 3, 6, and $12 \mathrm{mg} / \mathrm{mL}$, and with EVs). Viscosity was measured with a $40 \mathrm{~mm}$ diameter aluminum Peltier plate at the different conditions (from three different batches, each sample in triplicate). The rheometer was maintained at $25^{\circ} \mathrm{C}$ and viscosity was measured between 0.1 and $500 \mathrm{~Hz}$. For storage $\left(\mathrm{G}^{\prime}\right)$ and loss modulus (G') measurements, $1500 \mu \mathrm{L}$ of hydrogel solution was gelled for $24 \mathrm{~h}$ at $37^{\circ} \mathrm{C}$ on $25 \mathrm{~mm}$ diameter disc-shaped molds. The linear viscoelastic region was determined in 3 samples, applying an oscillatory strain sweep with amplitudes from $0.01 \%$ to $200 \%$ at a frequency of $1 \mathrm{~Hz}$ using a $25 \mathrm{~mm}$ diameter sand-blasted Peltier plate. In 4 samples of each condition (from two different batches), the dynamic frequency sweep at a fixed strain within the viscoelastic region $(0.15 \%)$ was performed from 0.01 to $2 \mathrm{~Hz}$ at $37^{\circ} \mathrm{C} \mathrm{G}^{\prime}$ and $\mathrm{G}^{\prime \prime}$ at $1 \mathrm{rad} / \mathrm{s}(0.16 \mathrm{~Hz})$ were plotted.

Injection forces are also important parameters for shear-thinning hydrogel characterization [14]. Injectability of the different preparations was confirmed through a commercially available Myostar injection catheter equipped with a $27 \mathrm{G}$ needle at the tip (Biosense Webster, Irvine, CA, USA). The force required for injecting the different solutions with a $1 \mathrm{~mL}$ Luer-lock syringe connected to the Myostar catheter was measured at an injection speed of $500 \mu \mathrm{L} / \mathrm{min}$ using a QTest 1 L Elite System (MTS, Artisan Technology Group, Champaign, IL, USA) and recorded using TestWorks Software. Measurements were performed at room temperature in 3 samples for each group (PBS, cECMH, cECMH with PEG at the different concentrations, and EVs-PEG-cECMH).

\subsection{Scanning Electron Microscopy}

To study the effect of PEG and EVs on the hydrogel structure and fiber diameter, SEM images from hydrogels of two different batches at the different conditions (cECMH, cECMH with PEG at 3, 6, and $12 \mathrm{mg} / \mathrm{mL}$, and EVs-PEG-cECMH) were taken. The solubilized matrix $(800 \mu \mathrm{L})$ was gelled at $37^{\circ} \mathrm{C}$ for $24 \mathrm{~h}$, and then dehydrated with a series of ethanol washes ( 2 h at $20 \%, 2$ h at $40 \%$, overnight at $60 \%, 2 \mathrm{~h}$ at $80 \%, 2 \mathrm{~h}$ at $100 \%$, and overnight at $100 \%$ ). The dehydrated samples were dried in a Thar R100W reactor by using supercritical $\mathrm{CO}_{2}$, as previously described [28], and then were immersed in liquid nitrogen before sectioning. Sectioned samples were mounted and sputter coated for $75 \mathrm{~s}$ with gold using Leica EM ACE600 (Wetzlar, Germany) to prepare them for imaging with a Philips XL30 SEM (Eindhoven, The Netherlands). Four random images at 24,000x were taken for each sample, and the widths of 20 distinguishable fibers per image (160 fibers per condition) were measured using ImageJ Software. 


\subsection{Enzymatic Degradation Assay}

The degradation assay of the hydrogels from six samples (coming from three different batches) at the different conditions ( was performed as previously described, with slight modifications [20]. Briefly, the solubilized matrix $(20 \mu \mathrm{L})$ was gelled at $37^{\circ} \mathrm{C}$ for $24 \mathrm{~h}$ in a $1.5 \mathrm{~mL}$ Eppendorf. A total of $20 \mu \mathrm{L}$ of collagenase type II (200 units/mL, LS004176, Worthington) dissolved in $0.1 \mathrm{M}$ Trizma base buffer (T1503, Sigma-Aldrich) pH 7.4 and $0.25 \mathrm{M} \mathrm{CaCl}_{2}$ were added, and the samples were further incubated at $37^{\circ} \mathrm{C}$ for 5,24 , and $48 \mathrm{~h}$. A total of $20 \mu \mathrm{L}$ of collagenase in $20 \mu \mathrm{L}$ of PBS was used as blanks. Following incubation, the samples were centrifuged at $15000 \mathrm{rpm}$ for $5 \mathrm{~min}$, and $10 \mu \mathrm{L}$ of the supernatant was mixed with an equal volume of $2 \%$ ninhydrin reagent solution (N7285, Sigma-Aldrich). The samples were boiled at $10 \mathrm{~min}$ in a water bath, and then $380 \mu \mathrm{L}$ of distilled water was added. In total, $100 \mu \mathrm{L}$ (in triplicate) was transferred into a 96-well plate and the OD at $570 \mathrm{~nm}$ was measured using an EMax ${ }^{\circledR}$ Plus Microplate Reader (BioteK, Winooski, VT, USA). With the ninhydrin assay, a higher OD at $570 \mathrm{~nm}$ is indicative of more soluble amines.

\subsection{EV Bioactivity-Antisenescent Effect}

The bioactivity of the EVs, the cECMH, and the PEG-CECMH with the encapsulated EVs (EVs-PEG-cECMH) was tested by investigating their anti-senescent effect in EDCs coming from two patients different from the EVs used as treatment. In triplicate, $250 \mu \mathrm{L}$ samples of EVs (at $0.56 \mathrm{mg} / \mathrm{mL}$ ) in SFM, cECM alone, or EVs in PEG-cECM were incubated for gelation during $24 \mathrm{~h}$ at $37^{\circ} \mathrm{C}$ in 12 -well Transwells ${ }^{\circledR}\left(3460\right.$, Corning $\left.{ }^{\circledR}\right)$. EDCs from passage 3 were seeded at a concentration of 15,000 cells $/ \mathrm{cm}^{2}$ in fibronectin precoated 12-well plates. The cells were left for $24 \mathrm{~h}$ to attach, and then the medium was changed to SFM ( $2 \mathrm{~mL} /$ well), and the Transwells ${ }^{\circledR}$ with SFM (controls), EVs in SFM, cECMH, or EVs-PEG-cECMH were added. After $72 \mathrm{~h}$, the Transwells ${ }^{\circledR}$ were removed and the cells fixed and stained with the senescence-associated beta-galactosidase assay, following the manufacturer's instructions (ab65351 Senescence Detection Kit, abcam ${ }^{\circledR}$ ). A total of 14 images at 20x (around 650 cells) per well were taken with a Leica DMI3000B optical microscope and Leica DFC310 FX camera (Wetzlar, Germany), and analyzed using ImageJ Software. Cells were classified as senescent or non-senescent depending on whether they presented a blue color, and the percentage of senescent cells was calculated.

\subsection{EV Release from cECMH}

Three samples of $100 \mu \mathrm{L}$ of solubilized cECM and with the EVs resuspended in PEGcECM were incubated for gelation during $24 \mathrm{~h}$ at $37^{\circ} \mathrm{C}$ in 24-well Transwells ${ }^{\circledR}(3422$, Corning $^{\circledR}$ ). EVs resuspended in SFM were used as controls. The samples were later placed in 24-well plates, where $600 \mu \mathrm{L}$ of SFM was added to each well. The medium was collected daily and replaced, and the number of particles released was measured in triplicate by NTA using a Nanosight NS300 instrument and Nanosight NTA 3.4. software (Malvern Paranalytical, Malvern, UK) [40]. The samples were injected at room temperature into the sample chamber using a syringe pump, and, for each sample, three videos of $60 \mathrm{~s}$ were recorded with screen gain 1 and camera level at 11 .

\subsection{EV In Vivo Retention \\ 4.10.1. EV Labeling}

Radioactive labeling of the isolated EVs was carried out with the SPECT radionuclide ${ }^{99 \mathrm{~m}} \mathrm{Tc}$ based on previous protocols of exosome radiolabeling [41]. Briefly, 9-12 mCi of commercial [ $\left.{ }^{99 \mathrm{~m}} \mathrm{Tc}\right] \mathrm{NaTcO}_{4}$ (Curium Pharma, Madrid, Spain) was reduced in the presence of $0.01 \mathrm{M} \mathrm{SnCl}_{2}$ in $\mathrm{HAc}(10 \%)$. The reaction was performed for $5 \mathrm{~min}$ at $37^{\circ} \mathrm{C}$ and $700 \mathrm{rpm}$, under $\mathrm{N}_{2}$ atmosphere. Then, the mixture was neutralized $(\mathrm{pH}=7)$ with $2.8 \mathrm{~N} \mathrm{NaOH}$. EVs $\left(3 \mathrm{mg}, 400 \mu \mathrm{L}\right.$ ) were added to the ${ }^{99 \mathrm{~m}} \mathrm{Tc}$ (IV) solution, and incubated and shacked for $30 \mathrm{~min}$ at $37^{\circ} \mathrm{C}$ and $700 \mathrm{rpm}$. Radiolabeled EVs were purified by centrifuging with $10 \mathrm{KDa}$ Amicon filters (Merck Life Science, Darmstadt, Germany) and resuspended in 
$400 \mu \mathrm{L}$ of PBS or PEG-cECM solution. Radiochemical purity of the recovered product was established by radio-thin-layer chromatography (iTLC) using a miniGita Single system (Elisa-Raytest, Angleur, Belgium).

\subsubsection{EV Tracking by SPECT-CT Imaging}

Multimodality imaging was performed with a small-animal SPECT scanner $(\mu \mathrm{SPECT}$, MILabs, Houten, the Netherlands) and a preclinical CT system (Super Argus, SEDECAL, Algete, Spain). SPECT and CT images were acquired $15 \mathrm{~min}$ and $24 \mathrm{~h}$ after subcutaneous administration of an average $300 \mu \mathrm{Ci}$ dose (no significant differences between groups) of radiolabeled EVs ( $3 \mathrm{mg}$ of protein, $400 \mu \mathrm{L}$ of PBS or PEG-cECM solution). Six Balb/C, 15 -week-old female mice of $23.3 \pm 1.8 \mathrm{~g}$ were divided in two groups; half of them $(n=3)$ received EVs in PBS and the rest EVs in PEG-cECM. To co-register the SPECT and CT images, each animal was placed on an in-house multimodal bed surrounded by three noncoplanar capillaries filled with a mixture of ${ }^{99 \mathrm{~m}} \mathrm{Tc}$ and Iopamiro (Bracco Imaging S.p.A, Milan, Italy), which was visible in both modalities. The SPECT acquisition parameters were an isotropic voxel size of $0.75 \mathrm{~mm}$ and an acquisition time of $15 \mathrm{~min}$ and $1.25 \mathrm{~h}$. SPECT images were reconstructed using two-dimensional ordered subset expectation maximization (OSEM-2D) with 16 subsets and 1 iteration. The $\mathrm{CT}$ was obtained immediately after completion of SPECT imaging. CT study was acquired using an X-ray beam current of $240 \mathrm{~mA}$ and a tube voltage of $40 \mathrm{kVp}$, and reconstructed using an FDK algorithm [42]. SPECT-CT images were co-registered following the method of García-Vazquez V. [43].

\subsubsection{Image Analysis}

The area of radioactivity was segmented on the SPECT images using a threshold at $50 \%$ of the maximum using Image $1.49 \mathrm{i}$. The area was compared between the animals that had been injected with the radioactively labeled EVs resuspended in PBS vs. EVs resuspended in the PEG-cECM.

\subsection{Statistical Analysis}

Results are presented as mean \pm standard deviation in the text and in figures. Continuous variables were compared using Student's $t$-tests. Pearson tests were performed to study the correlation between the different parameters and the PEG concentration in the $\mathrm{CECMH}$.

\section{Conclusions}

Hydrogels derived from cardiac extracellular matrix (CECMH) incorporating polyethylene glycol (PEG) present promising characteristics for the delivery of extracellular vesicles (EVs) for different regenerative applications. Once injected into the target tissue, the product rapidly gels at physiological temperature. The incorporation of EVs with PEG to the cECMH improves the mechanical properties while maintaining the injectability and the biodegradability. In addition, the combined product showed bioactivity similar to its individual components. With the EVs-PEG-cECMH, the EVs are better retained in vivo on-site. These improved properties of the combined product solve some of the current limitations of the individual use of these regenerative components, which may be translated into an increased therapeutic efficacy.

Author Contributions: Conceptualization, L.G.-C. and L.G.-S.; methodology, L.G.-C. and L.G.-S.; formal analysis, L.G.-C. and L.G.-S.; investigation, L.G.-C., M.L.L.-D., D.V., V.M., M.I.G., S.B.B., J.S., and E.A.; resources, M.L.L.-D., D.V., B.S., L.C., Á.G., C.E. and R.B.; writing-original draft preparation, L.G.-C. and L.G.-S.; writing-review and editing, M.L.L.-D., D.V., M.I.G., B.S., L.C., Á.G., S.B.B., M.E.F.-S., C.E., J.S., E.A. and F.F.-A.; visualization, L.G.-C.; supervision, L.G.-S.; project administration, L.G.-S. and F.F.-A.; funding acquisition, F.F.-A. All authors have read and agreed to the published version of the manuscript.

Funding: This study was supported by the Instituto de Salud Carlos III, Ministerio de Ciencia e Innovación, Spain. PI16/01123; PI19/00161; Red de Terapia Celular (RD16.0011.0029) and CIBERCV 
(CB16.11.00292). It has also been partially supported by Comunidad de Madrid, projects S2017/BMD3867 and EXOHEP-CM S2017/BMD-3727, co-funded by European Structural and Investment Fund, FSE, to RB and by Instituto de Salud Carlos III through the project PT20/00044, co-funded by European Regional Development Fund "A way to make Europe".

Institutional Review Board Statement: The study was conducted according to the guidelines of the Declaration of Helsinki. Human cardiac biopsy collection for EDC and EV production was approved by the Ethics Committee Comité de Ética de Investigación con Medicamentos del Hospital General Universitario Gregorio Marañón (protocol code 331/16 and date of approval 15 December 2016). Pig heart collection for decellularization and procedures for retention studies in mice were approved by the Ethics Committee Comité de Ética de Experimentación Animal del Hospital Gregorio Marañón and by the Competent Authority (protocol code 115/15 approved in 2015, and protocol code 097/16 approved in 2016 respectively).

Informed Consent Statement: Informed consent was obtained from all subjects involved in the study.

Acknowledgments: We kindly thank Luis Rodríguez for their guidance with the injectability and rheological experiments and Cristina Moral for her technical assistance with the SEM. We also thank Alexandra de Francisco, Yolanda Sierra and María de la Jara Felipe for their excellent work with the animal preparation and imaging.

Conflicts of Interest: The authors declare no conflict of interest. The funders had no role in the design of the study; in the collection, analyses, or interpretation of data; in the writing of the manuscript, or in the decision to publish the results.

\section{References}

1. Povsic, T.J.; Sanz-Ruiz, R.; Climent, A.M.; Bolli, R.; Taylor, D.A.; Gersh, B.J.; Menasché, P.; Perin, E.C.; Pompilio, G.; Atsma, D.E.; et al. Reparative Cell Therapy for the Heart: Critical Internal Appraisal of the Field in Response to Recent Controversies. ESC Heart Fail. 2021, 8, 2306-2309. [CrossRef] [PubMed]

2. Madonna, R.; Van Laake, L.W.; Davidson, S.M.; Engel, F.B.; Hausenloy, D.J.; Lecour, S.; Leor, J.; Perrino, C.; Schulz, R.; Ytrehus, K.; et al. Position Paper of the European Society of Cardiology Working Group Cellular Biology of the Heart: Cell-Based Therapies for Myocardial Repair and Regeneration in Ischemic Heart Disease and Heart Failure. Eur. Heart J. 2016, 37, 1789-1798. [CrossRef] [PubMed]

3. Ibrahim, A.G.-E.; Cheng, K.; Marbán, E. Exosomes as Critical Agents of Cardiac Regeneration Triggered by Cell Therapy. Stem Cell Rep. 2014, 2, 606-619. [CrossRef] [PubMed]

4. Chen, B.; Li, Q.; Zhao, B.; Wang, Y. Stem Cell-Derived Extracellular Vesicles as a Novel Potential Therapeutic Tool for Tissue Repair. Stem Cells Transl. Med. 2017, 6, 1753-1758. [CrossRef]

5. Gallet, R.; Dawkins, J.; Valle, J.; Simsolo, E.; de Couto, G.; Middleton, R.; Tseliou, E.; Luthringer, D.; Kreke, M.; Smith, R.R.; et al. Exosomes Secreted by Cardiosphere-Derived Cells Reduce Scarring, Attenuate Adverse Remodelling, and Improve Function in Acute and Chronic Porcine Myocardial Infarction. Eur. Heart J. 2017, 38, 201-211. [CrossRef]

6. Grigorian-Shamagian, L.; Liu, W.; Fereydooni, S.; Middleton, R.C.; Valle, J.; Cho, J.H.; Marbán, E. Cardiac and Systemic Rejuvenation after Cardiosphere-Derived Cell Therapy in Senescent Rats. Eur. Heart J. 2017, 38, 2957-2967. [CrossRef]

7. Gómez-Cid, L.; Moro-López, M.; de la Nava, A.S.; Hernández-Romero, I.; Fernández, A.I.; Suárez-Sancho, S.; Atienza, F.; Grigorian-Shamagian, L.; Fernández-Avilés, F. Electrophysiological Effects of Extracellular Vesicles Secreted by CardiosphereDerived Cells: Unraveling the Antiarrhythmic Properties of Cell Therapies. Processes 2020, 8, 924. [CrossRef]

8. Kennedy, T.L.; Russell, A.J.; Riley, P. Experimental Limitations of Extracellular Vesicle-Based Therapies for the Treatment of Myocardial Infarction. Trends Cardiovasc. Med. 2020. [CrossRef]

9. Seif-Naraghi, S.B.; Singelyn, J.M.; Salvatore, M.A.; Osborn, K.G.; Wang, J.J.; Sampat, U.; Kwan, O.L.; Strachan, G.M.; Wong, J.; Schup-Magoffin, P.J.; et al. Safety and Efficacy of an Injectable Extracellular Matrix Hydrogel for Treating Myocardial Infarction. Sci. Transl. Med. 2013, 5, 173ra25. [CrossRef]

10. Hernandez, M.J.; Christman, K.L. Designing Acellular Injectable Biomaterial Therapeutics for Treating Myocardial Infarction and Peripheral Artery Disease. JACC Basic Transl. Sci. 2017, 2, 212-226. [CrossRef]

11. Peña, B.; Laughter, M.; Jett, S.; Rowland, T.J.; Taylor, M.R.G.; Mestroni, L.; Park, D. Injectable Hydrogels for Cardiac Tissue Engineering. Macromol. Biosci. 2018, 18, e1800079. [CrossRef]

12. Chen, P.; Wang, L.; Fan, X.; Ning, X.; Yu, B.; Ou, C.; Chen, M. Targeted Delivery of Extracellular Vesicles in Heart Injury. Theranostics 2021, 11, 2263-2277. [CrossRef]

13. Hoeeg, C.; Dolatshahi-Pirouz, A.; Follin, B. Injectable Hydrogels for Improving Cardiac Cell Therapy-In Vivo Evidence and Translational Challenges. Gels 2021, 7, 7. [CrossRef]

14. Chen, M.H.; Wang, L.L.; Chung, J.J.; Kim, Y.-H.; Atluri, P.; Burdick, J.A. Methods to Assess Shear-Thinning Hydrogels for Application as Injectable Biomaterials. ACS Biomater. Sci. Eng. 2017, 3, 3146-3160. [CrossRef] [PubMed] 
15. Chen, C.W.; Wang, L.L.; Zaman, S.; Gordon, J.; Arisi, M.F.; Venkataraman, C.M.; Chung, J.J.; Hung, G.; Gaffey, A.C.; Spruce, L.A.; et al. Sustained Release of Endothelial Progenitor Cell-Derived Extracellular Vesicles from Shear-Thinning Hydrogels Improves Angiogenesis and Promotes Function after Myocardial Infarction. Cardiovasc. Res. 2018, 114, 1029-1040. [CrossRef] [PubMed]

16. Han, C.; Zhou, J.; Liang, C.; Liu, B.; Pan, X.; Zhang, Y.; Wang, Y.; Yan, B.; Xie, W.; Liu, F.; et al. Human Umbilical Cord Mesenchymal Stem Cell Derived Exosomes Encapsulated in Functional Peptide Hydrogels Promote Cardiac Repair. Biomater. Sci. 2019, 7, 2920-2933. [CrossRef] [PubMed]

17. Waters, R.; Alam, P.; Pacelli, S.; Chakravarti, A.R.; Ahmed, R.P.H.; Paul, A. Stem Cell-Inspired Secretome-Rich Injectable Hydrogel to Repair Injured Cardiac Tissue. Acta Biomater. 2018, 69, 95-106. [CrossRef]

18. Johnson, T.D.; Lin, S.Y.; Christman, K.L. Tailoring Material Properties of a Nanofibrous Extracellular Matrix Derived Hydrogel. Nanotechnology 2011, 22, 494015. [CrossRef]

19. Gobeaux, F.; Mosser, G.; Anglo, A.; Panine, P.; Davidson, P.; Giraud-Guille, M.-M.; Belamie, E. Fibrillogenesis in Dense Collagen Solutions: A Physicochemical Study. J. Mol. Biol. 2008, 376, 1509-1522. [CrossRef]

20. Grover, G.N.; Rao, N.; Christman, K.L. Myocardial Matrix-Polyethylene Glycol Hybrid Hydrogels for Tissue Engineering. Nanotechnology 2014, 25, 014011. [CrossRef]

21. Suri, S.; Schmidt, C.E. Photopatterned Collagen-Hyaluronic Acid Interpenetrating Polymer Network Hydrogels. Acta Biomater. 2009, 5, 2385-2397. [CrossRef] [PubMed]

22. Sargeant, T.D.; Desai, A.P.; Banerjee, S.; Agawu, A.; Stopek, J.B. An in Situ Forming Collagen-PEG Hydrogel for Tissue Regeneration. Acta Biomater. 2012, 8, 124-132. [CrossRef] [PubMed]

23. Engler, A.J.; Griffin, M.A.; Sen, S.; Bönnemann, C.G.; Sweeney, H.L.; Discher, D.E. Myotubes Differentiate Optimally on Substrates with Tissue-like Stiffness: Pathological Implications for Soft or Stiff Microenvironments. J. Cell Biol. 2004, 166, 877-887. [CrossRef] [PubMed]

24. Evans, N.D.; Gentleman, E. The Role of Material Structure and Mechanical Properties in Cell-Matrix Interactions. J. Mater. Chem. B 2014, 2, 2345-2356. [CrossRef]

25. Martens, T.P.; Godier, A.F.G.; Parks, J.J.; Wan, L.Q.; Koeckert, M.S.; Eng, G.M.; Hudson, B.I.; Sherman, W.; Vunjak-Novakovic, G. Percutaneous Cell Delivery into the Heart Using Hydrogels Polymerizing In Situ. Cell Transplant. 2009, 18, 297-304. [CrossRef]

26. Zhang, Y.; Kumar, P.; Lv, S.; Xiong, D.; Zhao, H.; Cai, Z.; Zhao, X. Recent Advances in 3D Bioprinting of Vascularized Tissues. Mater. Des. 2021, 199, 109398. [CrossRef]

27. Hernandez, M.J.; Gaetani, R.; Pieters, V.M.; Ng, N.W.; Chang, A.E.; Martin, T.R.; van Ingen, E.; Mol, E.A.; Dzieciatkowska, M.; Hansen, K.C.; et al. Decellularized Extracellular Matrix Hydrogels as a Delivery Platform for MicroRNA and Extracellular Vesicle Therapeutics. Adv. Ther. 2018, 1, 1800032. [CrossRef]

28. Montero, A.; Acosta, S.; Hernández, R.; Elvira, C.; Jorcano, J.L.; Velasco, D. Contraction of Fibrin-Derived Matrices and Its Implications for in Vitro Human Skin Bioengineering. J. Biomed. Mater. Res. A 2021, 109, 500-514. [CrossRef]

29. Reing, J.E.; Zhang, L.; Myers-Irvin, J.; Cordero, K.E.; Freytes, D.O.; Heber-Katz, E.; Bedelbaeva, K.; McIntosh, D.; Dewilde, A.; Braunhut, S.J.; et al. Degradation Products of Extracellular Matrix Affect Cell Migration and Proliferation. Tissue Eng. Part. A 2009, 15, 605-614. [CrossRef]

30. Li, F.; Li, W.; Johnson, S.; Ingram, D.; Yoder, M.; Badylak, S. Low-Molecular-Weight Peptides Derived from Extracellular Matrix as Chemoattractants for Primary Endothelial Cells. Endothelium 2004, 11, 199-206. [CrossRef] [PubMed]

31. Annabi, N.; Nichol, J.W.; Zhong, X.; Ji, C.; Koshy, S.; Khademhosseini, A.; Dehghani, F. Controlling the Porosity and Microarchitecture of Hydrogels for Tissue Engineering. Tissue Eng. Part B Rev. 2010, 16, 371-383. [CrossRef]

32. Sánchez, P.L.; Fernández-Santos, M.E.; Costanza, S.; Climent, A.M.; Moscoso, I.; Gonzalez-Nicolas, M.A.; Sanz-Ruiz, R.; Rodríguez, H.; Kren, S.M.; Garrido, G.; et al. Acellular Human Heart Matrix: A Critical Step toward Whole Heart Grafts. Biomaterials 2015, 61, 279-289. [CrossRef]

33. Izquierdo, I.; Rosa, I.; Bravo, S.B.; Guitián, E.; Pérez-Serra, A.; Campuzano, O.; Brugada, R.; Mangas, A.; García, Á.; Toro, R. Proteomic Identification of Putative Biomarkers for Early Detection of Sudden Cardiac Death in a Family with a LMNA Gene Mutation Causing Dilated Cardiomyopathy. J. Proteom. 2016, 148, 75-84. [CrossRef] [PubMed]

34. Peñas-Martínez, J.; Barrachina, M.N.; Cuenca-Zamora, E.J.; Luengo-Gil, G.; Bravo, S.B.; Caparrós-Pérez, E.; Teruel-Montoya, R.; Eliseo-Blanco, J.; Vicente, V.; García, Á.; et al. Qualitative and Quantitative Comparison of Plasma Exosomes from Neonates and Adults. Int. J. Mol. Sci. 2021, 22, 1926. [CrossRef]

35. Bonzon-Kulichenko, E.; Pérez-Hernández, D.; Núñez, E.; Martínez-Acedo, P.; Navarro, P.; Trevisan-Herraz, M.; del Carmen Ramos, M.; Sierra, S.; Martínez-Martínez, S.; Ruiz-Meana, M.; et al. A Robust Method for Quantitative High-Throughput Analysis of Proteomes by 18 O Labeling. Mol. Cell. Proteom. 2011, 10, M110.003335. [CrossRef]

36. Perez-Hernandez, D.; Gutiérrez-Vázquez, C.; Jorge, I.; López-Martín, S.; Ursa, A.; Sánchez-Madrid, F.; Vázquez, J.; Yáñez-Mó, M The Intracellular Interactome of Tetraspanin-Enriched Microdomains Reveals Their Function as Sorting Machineries toward Exosomes. J. Biol. Chem. 2013, 288, 11649-11661. [CrossRef] [PubMed]

37. Shilov, I.V.; Seymour, S.L.; Patel, A.A.; Loboda, A.; Tang, W.H.; Keating, S.P.; Hunter, C.L.; Nuwaysir, L.M.; Schaeffer, D.A. The Paragon Algorithm, a next Generation Search Engine That Uses Sequence Temperature Values and Feature Probabilities to Identify Peptides from Tandem Mass Spectra. Mol. Cell. Proteom. 2007, 6, 1638-1655. [CrossRef] [PubMed] 
38. Tang, W.H.; Shilov, I.V.; Seymour, S.L. Nonlinear Fitting Method for Determining Local False Discovery Rates from Decoy Database Searches. J. Proteome Res. 2008, 7, 3661-3667. [CrossRef] [PubMed]

39. Singelyn, J.M.; DeQuach, J.A.; Seif-Naraghi, S.B.; Littlefield, R.B.; Schup-Magoffin, P.J.; Christman, K.L. Naturally Derived Myocardial Matrix as an Injectable Scaffold for Cardiac Tissue Engineering. Biomaterials 2009, 30, 5409-5416. [CrossRef]

40. Filipe, V.; Hawe, A.; Jiskoot, W. Critical Evaluation of Nanoparticle Tracking Analysis (NTA) by NanoSight for the Measurement of Nanoparticles and Protein Aggregates. Pharm. Res. 2010, 27, 796-810. [CrossRef] [PubMed]

41. González, M.I.; Martín-Duque, P.; Desco, M.; Salinas, B. Radioactive Labeling of Milk-Derived Exosomes with 99mTc and In Vivo Tracking by SPECT Imaging. Nanomaterials 2020, 10, 1062. [CrossRef] [PubMed]

42. Abella, M.; Vaquero, J.J.; Sisniega, A.; Pascau, J.; Udías, A.; García, V.; Vidal, I.; Desco, M. Software Architecture for Multi-Bed FDK-Based Reconstruction in X-Ray CT Scanners. Comput. Methods Programs Biomed. 2012, 107, 218-232. [CrossRef] [PubMed]

43. García-Vázquez, V.; Cussó, L.; Chamorro-Servent, J.; Mirones, I.; García-Castro, J.; López-Sánchez, L.; Peña-Zalbidea, S.; Montesinos, P.; Chavarrías, C.; Pascau, J.; et al. Registration of Small-Animal SPECT/MRI Studies for Tracking Human Mesenchymal Stem Cells. In Proceedings of the XIII Mediterranean Conference on Medical and Biological Engineering and Computing 2013, Seville, Spain, 25-28 September 2013; Roa Romero, L.M., Ed.; Springer International Publishing: Cham, Switzerland, 2014; pp. 399-402. 